\title{
Area postrema syndrome: a neurological presentation of nausea, vomiting and hiccups
}

\author{
Ka Hong Chan (10),' Galina Vorobeychik ${ }^{2}$
}

'Department of Medicine, Division of General Internal Medicine, The University of British Columbia Faculty of Medicine, Vancouver, British Columbia, Canada

${ }^{2}$ Department of Medicine, Division of Neurology, The University of British Columbia Faculty of Medicine, Burnaby, British Columbia, Canada

Correspondence to Dr Galina Vorobeychik; Galina.Vorobeychik@ fraserhealth.ca

Accepted 24 September 2020

Check for updates

(c) BMJ Publishing Group Limited 2020. No commercial re-use. See rights and permissions. Published by BMJ.

\begin{tabular}{l}
\hline To cite: Chan KH, \\
Vorobeychik G. BMJ Case \\
Rep 2020;13:e238588. \\
doi:10.1136/bcr-2020- \\
238588 \\
\hline
\end{tabular}

\section{DESCRIPTION}

A 44-year-old, previously healthy, Asian woman presented with a 1-month history of daily, intractable nausea, vomiting, hiccups and a $7 \mathrm{~kg}$ weight loss. She had persistent dry heaves, with food triggering vomiting. She had no neurological symptoms, including a normal examination and subsequent visual evoked potentials. Thorough medical investigations including haematology, biochemistry (including C-reactive protein) and autoimmune workup, CT and ultrasound abdominal imaging, as well as upper endoscopy were all normal, except for a positive anti-Sjögren's-syndrome-related antigen A autoantibodies (anti-SSA). She eventually underwent an encephalic and spinal MRI, demonstrating an isolated T2-weighted-Fluid-Attenuated Inversion Recovery (T2-FLAIR) enhancement in area postrema (figure 1). Although her aquaporin-4 IgG (AQP4-IgG) and antimyelin oligodendrocyte glycoprotein (MOG) antibodies were negative, her classical gastrointestinal symptoms combined with her MRI scan was highly suggestive of area postrema syndrome (APS), a characteristic presentation of neuromyelitis optica (NMO). ${ }^{1}$ Following treatment with pulse methylprednisolone, her symptoms recovered completely with subsequent weight recovery. This was followed by rituximab maintenance therapy with no recurrences for over 9 months.

$\mathrm{NMO}$ is an autoimmune, demyelinating disorder characterised by AQP4-IgG antibodies that more commonly affects women. Classical NMO is typically recognised by recurrent attacks of optic neuritis or transverse myelitis. Yet, as with our case, approximately $30 \%$ of patients can present with isolated brainstem syndromes, with the most common
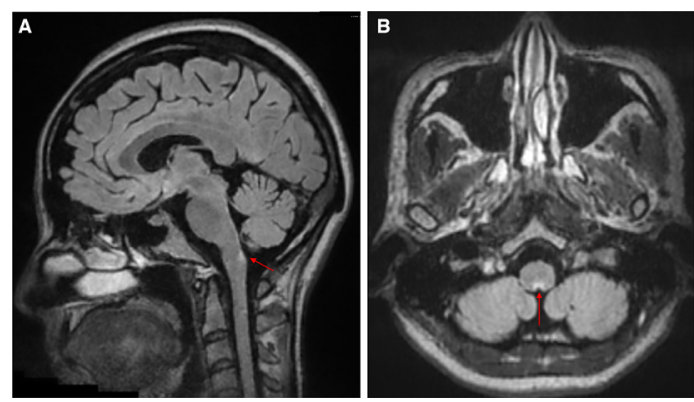

Figure 1 MRI T2 FLAIR sequence demonstrating bilateral lesions in the area postrema (highlighted by arrows) in the (A) sagittal and (B) transverse planes. Hyperintensity in this area at the level of the medulla oblongata with associated intractable nausea and vomiting is consistent with a diagnosis of area postrema syndrome.
Box 1 International consensus on the

diagnostic criteria of neuromyelitis optica spectrum disorders (NMOSD). Adapted from 9. AQP4: aquaporin-4, IgG: immunoglobulin G, LETM: longitudinally extensive transverse myelitis lesions

Diagnostic criteria for NMOSD with AQP4-lgG:

1. At least one core clinical characteristic (see below)

2. Positive AQP4-IgG

3. Exclusion of other diagnoses

Diagnostic criteria for NMOSD without or unknown AQP4-lgG:

1. At least two clinical characteristics occurring as a result of one or more clinical attacks and meeting all the below:

- At least one clinical characteristic must be optic neuritis, acute myelitis with LETM or area postrema syndrome

- Dissemination in space (two or more different core clinical characteristics)

- Fulfilment of additional MRI criteria, as applicable (see below)

2. Negative tests $A Q P 4-\lg G$ using the best available detection method, or testing unavailable

3. Exclusion of other diagnoses

Core clinical characteristics:

1. Optic neuritis

2. Acute myelitis

3. Area postrema syndrome

4. Acute brain stem syndrome

5. Symptomatic narcolepsy or acute diencephalic clinical syndrome with NMOSD-typical diencephalic MRI lesions

6. Symptomatic cerebral syndrome with NMOSDtypical brain lesions

Additional MRI requirements for NMOSD without or unknown AQP4-lgG:

1. Acute optic neuritis requires brain MRI showing (a) normal findings or only nonspecific white matter lesions, OR (b) optic nerve MRI with T2hyperintense lesion or T1-weighted gadoliniumenhancing lesion extending over $>1 / 2$ optic nerve length or involving optic chiasm

2. Acute myelitis requires associated intramedullary MRI lesion extending $\geq 3$ contiguous segments (LETM) $O R \geq 3$ contiguous segments of focal spinal cord atrophy in patients with history compatible with acute myelitis

3. Area postrema syndrome requires associated dorsal medulla/area postrema lesions 


\section{Box 1}

4. Acute brain stem syndrome requires associated periependymal brainstem lesions

Box originally from Wingerchuk et al. ${ }^{9}$

symptoms being intractable nausea, vomiting and hiccups due to APS. $^{23}$ This has been shown to be more common in the Asian and African-American populations. ${ }^{4}$

APS is characterised by lesions found in the area postrema (AP), an area rich in AQP4 receptors. Anatomically, AP is a vascular structure found in the floor of the fourth ventricle, and act as the vomiting centre by combining chemical and neural inputs from blood and brainstem, respectively. ${ }^{5}$ The leaky blood-brain barrier found here makes it accessible to AQP4-IgG, and is thought to represent an early phase of $\mathrm{NMO}{ }^{3}$ Unlike spinal and optic lesions of classic NMO, lesions in the AP usually demonstrate more inflammation than demyelination and necrosis, explaining the potential for complete recovery following treatment. ${ }^{12}$ Binding of IgG to AQP4 in the AP lacks immunoreactivity to activate the

\section{Patient's perspective}

I had intractable nausea, vomiting, and hiccups for a long time. I consulted several doctors in different places, but I did not get better. I am very thankful to whole team of doctors and hospital staff for appropriate treatment and care. After I was treated with steroids and Rituximab, nausea, vomiting and hiccups disappeared.

\section{Learning points}

Area postrema syndrome (APS) is part of the neuromyelitis optica spectrum of disorders, and can present solely with nausea, vomiting and hiccups and no neurological symptoms.

- Many cases of APS can present atypically with aquaporin-4 antibody negativity, with lesions on MRI in the area postrema, an emetogenic centre targeted by the antibodies, being highly suggestive of this disorder.

- Prompt treatment with steroids followed by maintenance therapy with rituximab can reduce the severe neurological morbidity and mortality of this disorder. complement system, and instead, causes receptor downregulation. The resulting alterations to neurotransmitter homeostasis trigger vomiting. ${ }^{1}$ Rarely in adults, external mass effect (eg, tumours) can also affect this area. ${ }^{6}$

Although the presence of anti-SSA is associated with serum AQP4-IgG positivity in the Asian population, it was negative in our case. ${ }^{7}$ Interestingly, only $14 \%$ of patients presenting with APS are. ${ }^{1}$ MOG positivity is also rare. ${ }^{8}$ T2 lesions within AP is characteristic of NMO as per the diagnostic criteria (box 1). ${ }^{9}$ Early recognition of this disease allows for prompt treatment that may reduce the morbidity and mortality of this severe syndrome, with $50 \%$ of patients having visual or ambulatory impairments within 5 years. ${ }^{9}$ Acute attacks are typically treated with pulse methylprednisolone for 3-5 days or plasma exchange. Maintenance therapy is initiated subsequently to reduce relapses. Azathioprine and rituximab followed by mycophenolate mofetil are the best studied, with some evidence suggesting rituximab to be the most effective. ${ }^{10}$

Contributors $\mathrm{KHC}$ wrote the initial manuscript. GV provided direction and editing of this manuscript.

Funding The authors have not declared a specific grant for this research from any funding agency in the public, commercial or not-for-profit sectors.

Competing interests None declared.

Patient consent for publication Obtained.

Provenance and peer review Not commissioned; externally peer reviewed.

\section{ORCID iD}

Ka Hong Chan http://orcid.org/0000-0002-5166-0653

\section{REFERENCES}

1 Iorio R, Lucchinetti CF, Lennon VA, et al. Intractable nausea and vomiting from autoantibodies against a brain water channel. Clin Gastroenterol Hepatol 2013;11:240-5.

2 Shosha E, Dubey D, Palace J, et al. Area postrema syndrome: frequency, criteria, and severity in AQP4-IgG-positive NMOSD. Neurology 2018;91:e1642-51.

3 Kremer L, Mealy M, Jacob A, et al. Brainstem manifestations in neuromyelitis optica: a multicenter study of 258 patients. Mult Scler 2014;20:843-7.

$4 \mathrm{Kim} \mathrm{S-H,} \mathrm{Mealy} \mathrm{MA,} \mathrm{Levy} \mathrm{M,} \mathrm{et} \mathrm{al.} \mathrm{Racial} \mathrm{differences} \mathrm{in} \mathrm{neuromyelitis} \mathrm{optica} \mathrm{spectrum}$ disorder. Neurology 2018;91:e2089-99.

5 Sarnat HB, Flores-Sarnat L, Boltshauser E. Area postrema: fetal maturation, tumors, vomiting center, growth, role in neuromyelitis optica. Pediatr Neurol 2019;94:21-31.

6 Zeiner PS, Brandhofe A, Müller-Eschner M, et al. Area postrema syndrome as frequent feature of Bickerstaff brainstem encephalitis. Ann Clin Trans/ Neurol 2018;5:5.

7 Park J-H, Hwang J, Min J-H, et al. Presence of anti-Ro/SSA antibody may be associated with anti-aquaporin-4 antibody positivity in neuromyelitis optica spectrum disorder. J Neurol Sci 2015:348:132-5.

8 Hyun J-W, Kwon YN, Kim S-M, et al. Value of area postrema syndrome in differentiating adults with AQP4 vs. MOG antibodies. Front Neurol 2020;11:396.

9 Wingerchuk DM, Banwell B, Bennett JL, et al. International consensus diagnostic criteria for neuromyelitis optica spectrum disorders. Neurology 2015;85:177-89.

10 Borisow N, Mori M, Kuwabara S, et al. Diagnosis and treatment of NMO spectrum disorder and MOG-Encephalomyelitis. Front Neurol 2018;9:888.

Copyright 2020 BMJ Publishing Group. All rights reserved. For permission to reuse any of this content visit

https://www.bmj.com/company/products-services/rights-and-licensing/permissions/

BMJ Case Report Fellows may re-use this article for personal use and teaching without any further permission.

Become a Fellow of BMJ Case Reports today and you can:

- Submit as many cases as you like

- Enjoy fast sympathetic peer review and rapid publication of accepted articles

- Access all the published articles

Re-use any of the published material for personal use and teaching without further permission

Customer Service

If you have any further queries about your subscription, please contact our customer services team on +44 (0) 2071111105 or via email at support@bmj.com.

Visit casereports.bmj.com for more articles like this and to become a Fellow 